record an alarming temperature reading, it indicates that the cell's internal temperatures have reached a point where no external controls can stop the thermal runaway. With the thermally sensitive new strain gauges, the changes in slope are so sensitive that this change can be used to stop the charge or remove the load on the lithium-ion cells before the event can spiral into an uncontrollable one.
Four different configurations of lithium-ion cells of were tested. Two cylindrical cells with metal containers (two different diameters), a prismatic metal container cell type, and a pouch cell type (aluminized plastic pouch) were used for the study. Several tests were performed on all our designs. The tests included normal charge and discharge cycling at two different charge and discharge rates at room temperature and at low temperature. The tests also included off-nominal conditions of overcharge, overdischarge, external short, heat-tovent, and crush (simulated internal short). Overcharge tests and external short tests provide the most valuable data as these conditions produce the worstcase reactions in lithium-ion cells.

This work was done by Judith Jeevarajan of Johnson Space Center. Further information is contained in a TSP (see page 1). MSC-24764-1

\title{
(3) In-Service Monitoring of Steam Pipe Systems at High Temperatures
}

\author{
This system can be used by utility companies for steam pipe systems incorporating \\ multiple manholes.
}

\author{
NASA's Jet Propulsion Laboratory, Pasadena, California
}

An effective, in-service health monitoring system is needed to track water condensation in real time through the walls of steam pipes. The system is required to measure the height of the condensed water from outside the pipe, while operating at temperatures that are as high as $250{ }^{\circ} \mathrm{C}$. The system needs to account for the effects of water flow and cavitation. In addition, it is desired that the system does not require perforating the pipes and thereby reducing the structural integrity.

Generally, steam pipes are used as part of the district heating system carrying steam from central power stations under the streets to heat, cool, or supply power to high-rise buildings and businesses. This system uses ultrasonic waves in pulse-echo and acquires reflected signal data. Via autocorrelation, it determines the water height while eliminating the effect of noise and multiple reflections from the wall of the pipe.

The system performs nondestructive monitoring through the walls of steam pipes, and automatically measures the height of condensed water while operating at the high-temperature conditions of $250{ }^{\circ} \mathrm{C}$. For this purpose, the ultrasonic pulse-echo method is used where the time-of-flight of the wave reflections inside the water are measured, and it is multiplied by the wave velocity to determine the height. The pulse-echo test consists of emitting ultrasonic wave pulses from a piezoelectric transducer and receiving the reflections from the top and bottom of the condensed water. A single transducer is used as a transmitter as well as the receiver of the ultrasonic waves. To

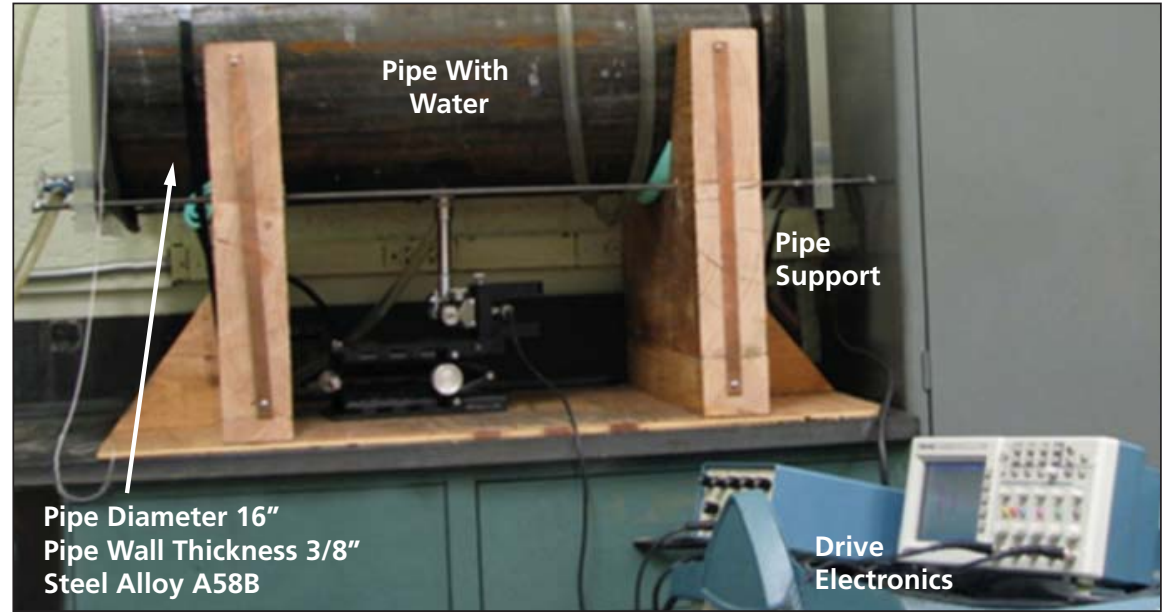

The testbed simulating the Steam Pipe and the in situ ultrasonic test setup.

obtain high resolution, a broadband transducer is used and the frequency can be in the range of 2.25 to $10 \mathrm{MHz}$, providing sharp pulses in the time domain allowing for higher resolution in identifying the individual reflections.

The pulse-echo transducer is connected to both the transmitter (function generator), which sends electric signals to generate the elastic wave, and the receiver, which amplifies the attenuated reflected waves that are converted to electric signals. To avoid damage to the receiver, the large signal from the generator is blocked by an electronic switching mechanism from reaching the receiving circuitry. To assure the operation of the transducer at the required temperature range, the piezoelectric transmitter/receiver is selected with a Curie temperature that is much higher. In addition, the system can be improved by introducing a heat sink between the transducer and the steam pipe, reducing the temperature requirements on the transducer.

This work was done by Yoseph Bar-Cohen, Shyh-Shiuh Lih, Mircea Badescu, Xiaoqi Bao, Stewart Sherrit, James S. Scott, Julian O. Blosiu, and Scott E. Widholm of Caltech for NASA's Jet Propulsion Laboratory. Further information is contained in a TSP (see page 1).

In accordance with Public Law 96-517, the contractor has elected to retain title to this invention. Inquiries concerning rights for its commercial use should be addressed to:

Innovative Technology Assets Management JPL

Mail Stop 202-233

4800 Oak Grove Drive

Pasadena, CA 91109-8099

E-mail: iaoffice@jpl.nasa.gov

Refer to NPO-47518, volume and number of this NASA Tech Briefs issue, and the page number. 